University of Massachusetts Amherst

ScholarWorks@UMass Amherst

\title{
Reconfiguring Gaussian Curvature of Hydrogel Sheets with Photoswitchable Host-Guest Interactions
}

\author{
Alexa S. Kuenstler \\ University of Massachusetts Amherst \\ Markus Lahikainen \\ Tampere University \\ Hantao Zhou \\ University of Massachusetts Amherst \\ Wenwen Xu \\ University of Massachusetts Amherst \\ Arri Priimagi \\ Tampere University
}

See next page for additional authors

Follow this and additional works at: https://scholarworks.umass.edu/muri_pubs

Kuenstler, Alexa S.; Lahikainen, Markus; Zhou, Hantao; Xu, Wenwen; Priimagi, Arri; and Hayward, Ryan C., "Reconfiguring Gaussian Curvature of Hydrogel Sheets with Photoswitchable Host-Guest Interactions" (2020). ACS Macro Letters. 15.

https://doi.org/10.1021/acsmacrolett.0c00469

This Article is brought to you for free and open access by the MURI on Photomechanical Materials at ScholarWorks@UMass Amherst. It has been accepted for inclusion in Publications by an authorized administrator of ScholarWorks@UMass Amherst. For more information, please contact scholarworks@library.umass.edu. 


\section{Authors}

Alexa S. Kuenstler, Markus Lahikainen, Hantao Zhou, Wenwen Xu, Arri Priimagi, and Ryan C. Hayward 


\title{
Reconfiguring Gaussian Curvature of Hydrogel Sheets with Photoswitchable Host-Guest Interactions
}

\author{
Alexa S. Kuenstler, ${ }^{\S}$ Markus Lahikainen, ${ }^{\S}$ Hantao Zhou, Wenwen Xu, Arri Priimagi,* \\ and Ryan C. Hayward*
}

Cite This: ACS Macro Lett. 2020, 9, 1172-1177

Read Online

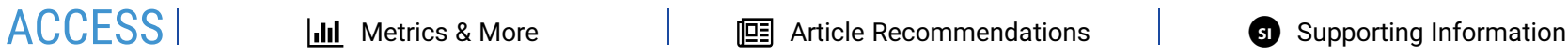

ABSTRACT: Photoinduced shape morphing has implications in fields ranging from soft robotics to biomedical devices. Despite considerable effort in this area, it remains a challenge to design materials that can be both rapidly deployed and reconfigured into multiple different three-dimensional forms, particularly in aqueous environments. In this work, we present a simple method to program and rewrite spatial variations in swelling and, therefore, Gaussian curvature in thin sheets of hydrogels using photoswitchable supramolecular complexation of azobenzene pendent groups with dissolved $\alpha$-cyclodextrin. We show that the extent of swelling can be programmed via the proportion of azobenzene isomers, with a $60 \%$ decrease in areal swelling from the all trans to the predominantly cis state near room temperature. The use of thin gel sheets provides fast response times in the range of a few tens of seconds, while the

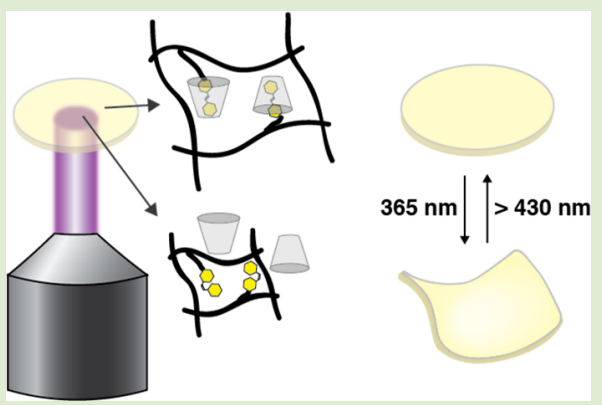
shape change is persistent in the absence of light thanks to the slow rate of thermal cis-trans isomerization. Finally, we demonstrate that a single gel sheet can be programmed with a first swelling pattern via spatially defined illumination with ultraviolet light, then erased with white light, and finally redeployed with a different swelling pattern.

$S_{\mathrm{r}}^{\mathrm{t}}$ timuli-responsive hydrogels offer diverse applications ranging from biomedical devices ${ }^{1}$ to soft actuators. ${ }^{2}$ By introducing inhomogeneous in-plane swelling profiles, thin gel sheets can be programmed to buckle into shapes with essentially arbitrary distributions of Gaussian curvature, providing great flexibility for the design of targeted three-dimensional (3D) shapes. ${ }^{3-5}$ However, the majority of work to date has focused on transformation through closely related families of shapes that are permanently programmed into the material through variations in cross-link density, ${ }^{6-10}$ alignment of anisotropic inclusions, ${ }^{11,12}$ or the presence of nonswelling components, ${ }^{13,14}$ and it remains a challenge to rationally design stimuli-responsive hydrogel platforms that are amenable to adopting multiple distinct 3D configurations. ${ }^{15,16}$

To enable rewritable shape changes, the incorporation of photoresponsive species offers an attractive means to prescribe complex morphogenesis with a high degree of spatiotemporal control. While photothermal moieties such as carbon materials $^{17-19}$ and gold nanoparticles ${ }^{20-25}$ have been exploited for photopatterned deswelling of thermoresponsive gels, the use of photochemical additives would offer advantages in terms of shape persistence and improved patterning resolution, due to the absence of thermal broadening and heat dissipation inherent to photothermal responses. ${ }^{26}$ Though photochemically addressable hydrogel systems are predicted to enable robust reconfigurable shape change, ${ }^{27,28}$ experimental demonstrations have been limited. The most promising approach to date has relied on spiropyran derivatives, where photoreversible ringopening and -closing reactions drive large changes in hydro- philicity and thus swelling. ${ }^{29-32}$ However, realization of robust photochemical responses has been complicated by narrow $\mathrm{pH}$ operational ranges and photoswitching fatigue in these systems.

Within the broader field of photoactive soft materials, azobenzene is among the most widely employed photoswitches because it isomerizes robustly over many cycles and is highly tunable in both its absorption characteristics and thermal relaxation kinetics. ${ }^{33}$ While azobenzene chromophores are routinely used in ordered polymer systems such as liquid crystal networks to impart large shape changes, ${ }^{34,35}$ their effect is typically less dramatic in hydrogels and dependent on how the photoswitch is incorporated. For example, azobenzene pendent groups have been shown to drive modest isomerization-induced increases in swelling ${ }^{36-38}$ due to the greater polarity of the cis isomer, ${ }^{39}$ while azobenzene cross-linkers yielded the opposite effect. $^{40,41}$

To enable photochemical shape morphing of hydrogels, we consider here reversible host-guest interactions with $\alpha$ cyclodextrin $(\alpha-\mathrm{CD})$, wherein trans-azobenzene inserts into the hydrophobic cavity of $\alpha$-CD while the cis-isomer is expelled. ${ }^{42}$ Using this scheme, azobenzene- and $\alpha$-CD-function-

Received: June 24, 2020

Accepted: July 22, 2020

Published: July 31, 2020 
alized hydrogels have been used to drive substantial volumetric changes ${ }^{4-46}$ through on-demand changes in cross-link density. However, this work has been limited to thick gels where moderate light penetration, slow cross-linking kinetics, and diffusion-limited mass transport result in modest shape changes. As an alternative approach, $\alpha$-CD complexes with transazobenzene are much more hydrophilic than free cisazobenzene, providing a simple way to modulate macromolecular hydrophilicity, an effect that has been widely exploited to tune the solubility of linear macromolecules ${ }^{4-49}$ but so far not employed for shape-programmable materials.

Herein, we introduce a facile approach to photoresponsive gels with rewritable Gaussian curvature by employing localized changes in hydrophobicity using azobenzene-functionalized poly ( $N$-isopropylacrylamide) (PNIPAm) gels containing free $\alpha$ CD units. Photoisomerization of azobenzene under UV light is used to impose a swelling pattern, and therefore a 3D shape, in thin gel sheets by breaking host-guest complexes. Subsequent exposure to visible light drives reisomerization to the transisomer and restoration of host-guest complexes, enabling a new 3D shape to be programmed through subsequent exposure to a different pattern of UV light, allowing for rapid and reconfigurable shape change.

Light-responsive gels are synthesized by copolymerization of $N$-isopropylacrylamide (NIPAm) and $N, N^{\prime}$-methylenebis(acrylamide) with 4-acrylamidoazobenzene (see Supporting Information for details) by free-radical polymerization to form poly(NIPAm-co-azobenzene) hydrogels (Scheme 1). Briefly,

Scheme 1. Synthesis of Light-Responsive Gels

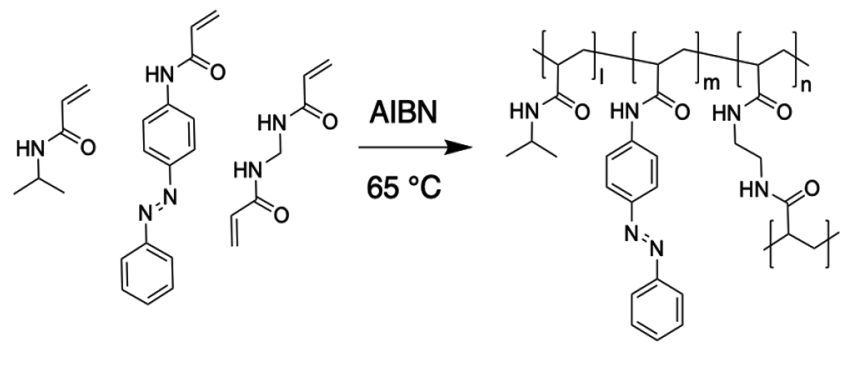

the monomer components are mixed with 5:1 tetrahydrofuran (THF): $\mathrm{H}_{2} \mathrm{O}$ and purged with nitrogen. Next, the pregel solution is infiltrated between two glass slides separated by $25 \mu \mathrm{m}$ and heated to $65{ }^{\circ} \mathrm{C}$ overnight to polymerize. Following synthesis, gels are swollen sequentially in fresh solutions of 5:1 THF: $\mathrm{H}_{2} \mathrm{O}$ and deionized $\mathrm{H}_{2} \mathrm{O}$ to remove unreacted monomers. After thermal equilibration in the dark, azobenzene units in the gel are in the trans form, and upon immersion in an aqueous solution of $15.4 \mathrm{mM}$ of $\alpha$-CD, gels are observed to rapidly (within tens of seconds) increase in area by $\approx 1.8$ times. This change in size is attributed to an increase in gel hydrophilicity as the hydrophobic inner cavity of $\alpha$-CD complexes with azobenzene via a hostguest interaction, leaving the hydrophilic outer portion of $\alpha$-CD to interface with the surrounding water. ${ }^{43}$

The photoresponsive properties of the gels are studied using UV-vis spectroscopy. Initially, azobenzene units in thermally equilibrated gels are in the trans form as indicated by the strong $\pi, \pi^{*}$ peak centered at $360 \mathrm{~nm}$ and a weak $\mathrm{n}, \pi^{*}$ at $430 \mathrm{~nm}$ (Figure 1A, black curve). Upon illumination with $50 \mathrm{~mW} \mathrm{~cm}^{-2}$ of 365 $\mathrm{nm}$ light, the azobenzene units are observed to switch from trans to $c i$ and achieve a photostationary state (PSS) of $>70 \%$ of $c i s$, as evidenced by a decrease in intensity and blue shift of the $\pi, \pi^{*}$
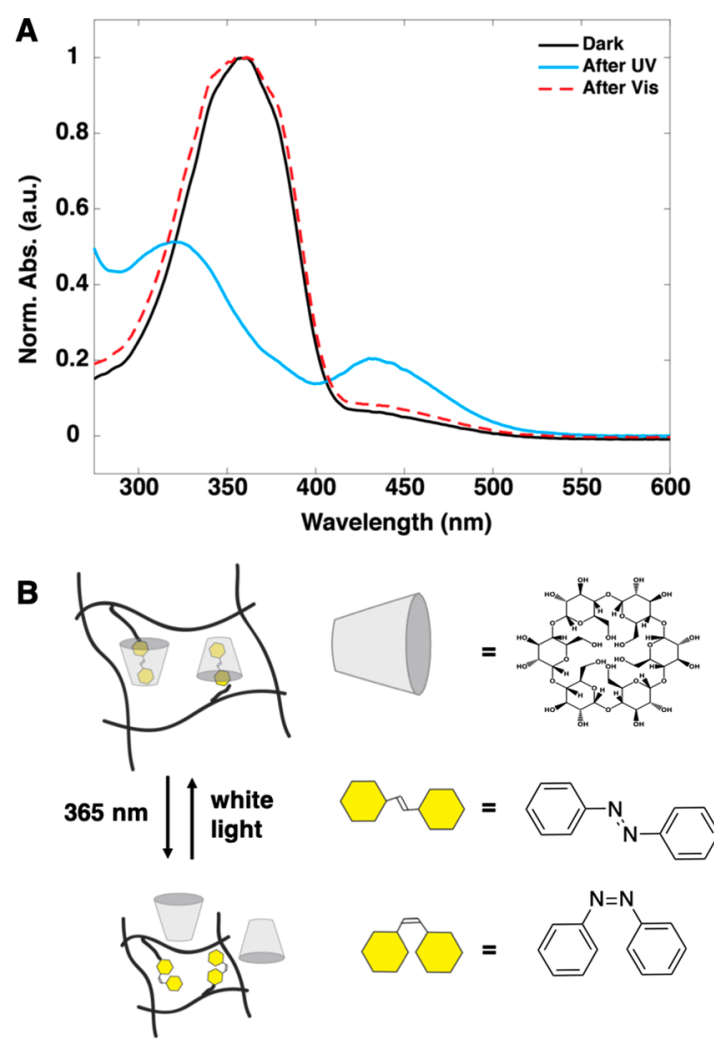

Figure 1. (A) UV-vis absorption spectra of the azobenzene-CD gel before (black) and after UV exposure (blue). The original state can be restored upon white-light exposure (red). (B) Schematic of reversible supramolecular complexation between azobenzene and $\alpha$-CD in thin gel sheets upon cis-trans isomerization, leading to reversible changes in swelling.

peak and an increase in intensity of the $n, \pi^{*}$ peak (Figure 1A, blue curve). While quantitative isomerization is limited by the overlap of the cis and trans spectra, the extinction coefficient at $365 \mathrm{~nm}$ decreases by a factor of 5 upon trans-cis isomerization (Figure S2). This results in an increase in the penetration depth of the gel at $365 \mathrm{~nm}$ from 13 to $67 \mu \mathrm{m}$, facilitating relatively uniform absorption through the sample thickness $(25 \mu \mathrm{m})$ and a high cis content at the PSS. Isomerization is accompanied by the decrease of $\alpha$-CD affinity for azobenzene due to the change in azobenzene geometry, which expels the chromophore from the $\alpha$-CD cavity (Figure 1B), as previously characterized by several groups. For example, in systems where both azobenzene and $\alpha$ $\mathrm{CD}$ are incorporated into polymer chains, the binding constant decreases from $2000 \mathrm{M}^{-1}$ to $35 \mathrm{M}^{-1}$ for $\alpha$-CD-trans-azobenzene and $\alpha$-CD-cis-azobenzene, respectively, ${ }^{50}$ though the exact binding constants can vary based on the chemical details. ${ }^{51}$ Additionally, isomerization is reversible by illumination with visible light (Figure 1A, red dashed curve), allowing for the formation and destruction of supramolecular complexes on demand.

The effect of photoisomerization on swelling is first investigated at room temperature under flood illumination with $50 \mathrm{~mW} \mathrm{~cm}^{-2}$ of $365 \mathrm{~nm}$ light. Prior to illumination, the gel is equilibrated in the dark to maximize trans-isomer content and is initially flat in the fully swollen state. When exposed to $365 \mathrm{~nm}$ light, the gel is observed to rapidly deswell, reaching equilibrium in $\approx 30 \mathrm{~s}$ (Video S1). The area of the gel following light exposure is reduced by $\approx 50 \%$ (Figure $2 \mathrm{~A}$ ), indicating that azobenzene isomerizes nearly uniformly through the thickness of the sample, 
A
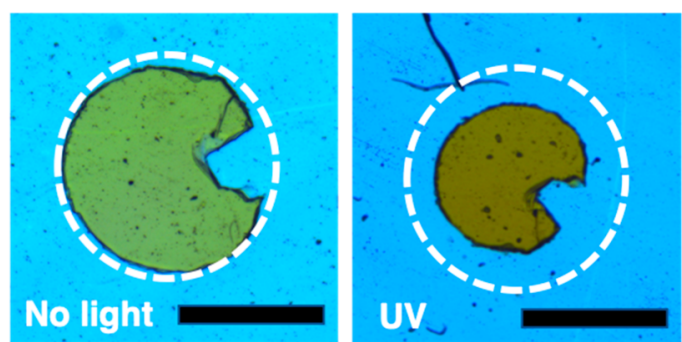

B

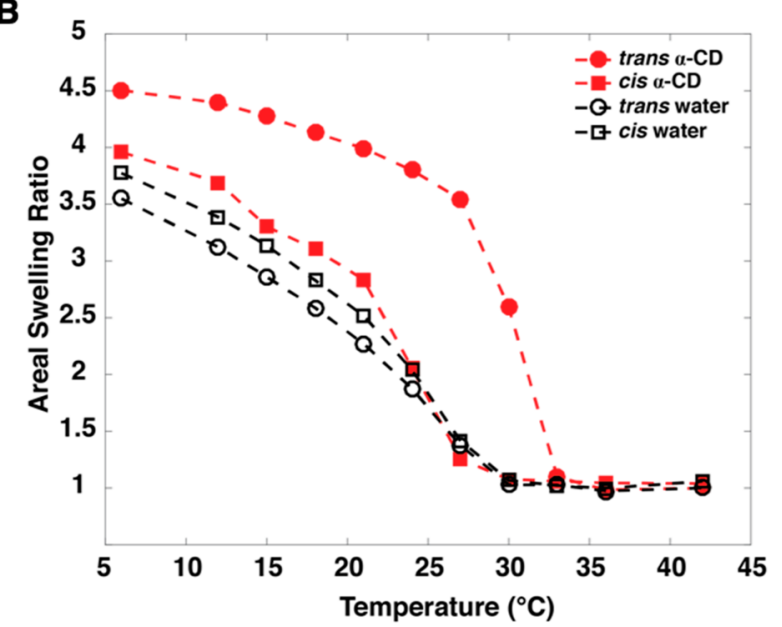

C

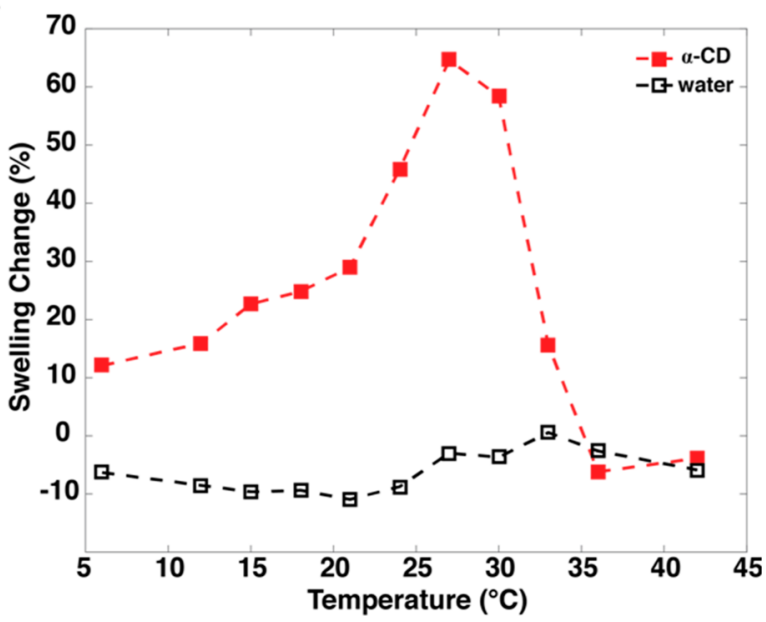

Figure 2. (A) Optical micrographs of gels with predominantly transazobenzene (left) and after photoisomerization with $365 \mathrm{~nm}$ light to $>70 \%$ cis-azobenzene (right) at room temperature. Scale bar: $1 \mathrm{~mm}$. (B) Equilibrium areal swelling ratios of hydrogels in pure water (black) and aqueous $\alpha$-CD solution (red) as a function of temperature under different illumination conditions. While increased cis content slightly increases the equilibrium swelling compared to trans in pure water, host-guest complexation of trans-azobenzene with $\alpha$-CD dramatically increases the swelling. (C) The percentage change in areal swelling for gels at the PSS compared to trans samples shows a maximum increase of $\approx 60 \%$, and a $50 \%$ increase near room temperature, in $\alpha$-CD solutions. Conversely, gels in deionized water show an $\approx 10 \%$ decrease in swelling near room temperature upon isomerization.

a consequence of the fact that the penetration depth at the PSS $(67 \mu \mathrm{m})$ is greater than the film thickness $(25 \mu \mathrm{m})$. Notably, the sample transiently bends during the initial stages of illumination before returning to a flat state (Figure S3), consistent with an isomerization-induced increase in penetration depth that ultimately allows for nearly uniform isomerization through the thickness of the gel at the PSS. Based on a typical poroelastic diffusion coefficient of $D=1-3 \times 10^{-11} \mathrm{~m}^{2} \mathrm{~s}^{-1,52}$ the characteristic diffusion time for this system can be estimated as $\tau \approx h^{2} D^{-1} \approx 20-60 \mathrm{~s}$, in close agreement with the deswelling time observed. However, this is also comparable to the time scale to reach the PSS for the light intensity used. Thus, the similar time scales coupled with the observation of transient bending under uniform illumination suggest that the deformation kinetics reflect contributions of both photoisomerization and mass transport.

To investigate how changes in isomer population-and thus supramolecular complexation-influence temperature-dependent swelling, gels are uniformly exposed to flood illumination in a bath of $\alpha$-CD solution $(15.4 \mathrm{mM})$ and held at a constant temperature ranging from 6 to $42{ }^{\circ} \mathrm{C}$ (Figure 2B,C). Areal swelling changes as a function of temperature and light exposure are measured by recording optical micrographs, and the areal swelling ratio is defined as the area of the gel at a given temperature normalized by the area of the gel at $42{ }^{\circ} \mathrm{C}$. Prior to illumination, gels equilibrated in the dark show a gradual deswelling upon heating to $26^{\circ} \mathrm{C}$, followed by a sharp transition and gradual deswelling to a constant area by $42{ }^{\circ} \mathrm{C}$ (Figure $2 \mathrm{~B}$, filled red circles). This behavior is consistent with the classical behavior of PNIPAm gels that display lower critical solution temperature (LCST) phase behavior. ${ }^{53}$ However, upon exposure to UV light, photoisomerization to a cis-rich PSS, and concomitant destruction of host-guest complexes dramatically alter the swelling characteristics due to the resulting increase in hydrophobicity that has been previously characterized by changes in water contact angle. ${ }^{54}$ Illumination with $365 \mathrm{~nm}$ light (Figure 2B, filled red squares) results in a reduced extent of swelling and a shift of the deswelling transition to lower temperatures with an increased breadth of the volume transition as the concentration of cis isomers increases and the azobenzene $-\alpha$-CD complexes are destroyed. This behavior is similar to how the choice of hydrophobic comonomer can be used to tune the swelling transition in nonphotoactive PNIPAm gels ${ }^{55}$ and is consistent with observations in linear poly(NIPAmco-azobenzene) polymers, where the LCST shifts to lower temperature upon decomplexation of azobenzene and cyclodextrin. ${ }^{56}$ We note that this change in swelling is due to the destruction of $\alpha$-CD complexes with trans-azobenzene and not simply due to changes in the trans-cis isomer population, which are nearly identical in both DI water and $\alpha$-CD solution (Figure S4). We verify this by control experiments in deionized water, which show only a small increase $(\approx 5 \%)$ in swelling upon transcis isomerization due to the moderate increase in polarity of the cis-isomer (Figure 2B, black curves; Figure 2C, black). ${ }^{39,57}$ Additionally, prior work has shown that $\alpha$-CD does not complex significantly with NIPAm side chains, ${ }^{58}$ and thus it is expected that the changes in swelling are primarily dictated by azobenzene-CD complexation. Notably, the change in swelling is maximized near room temperature, with an increase in inplane areal swelling by $50-60 \%$ relative to samples in the trans state (Figure 2C, red), making these materials well-suited for deployment at ambient temperatures. We note that while deformation of azo-based materials is often driven by a combination of photochemical and photothermal effects ${ }^{59,60}$ efficient photoisomerization as monitored by UV-vis and the persistence of deswelling upon the removal of illumination indicate that volume changes in our system are due to photochemical-and not photothermal-effects. 

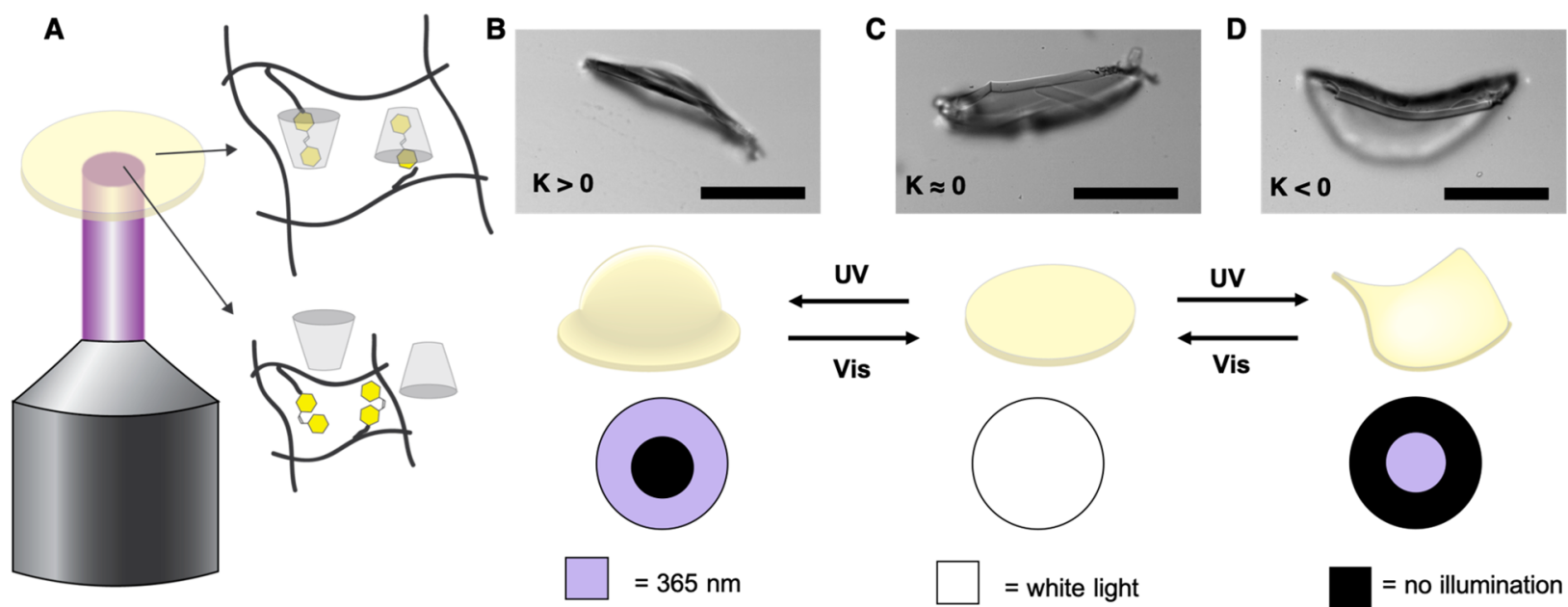

Figure 3. (A) Maskless lithography is used to locally pattern azobenzene isomerization-and therefore supramolecular complexation with $\alpha$-CDusing patterns of UV light. The resulting differences in gel swelling provide a programmed shape change with Gaussian curvature $K$ that can be erased by subsequent exposure to white light and then reprogrammed using another pattern of UV light. (B-D) Photographs of gels (top), cartoon schematic (middle), and corresponding illumination pattern (bottom). Scale bar: $1 \mathrm{~mm}$.

To probe the utility of photoinduced deswelling in shape morphing, gels are illuminated with $50 \mathrm{~mW} \mathrm{~cm} \mathrm{~cm}^{-2} \mathrm{UV}$ light patterned by a digital micromirror array and projected onto an immersed sample through an inverted microscope objective (Figure 3A). When $365 \mathrm{~nm}$ light is patterned in a circular annulus and projected onto a gel disk such that the center remains unilluminated, deswelling at the edges results in buckling out-of-plane into a spherical cap-like shape with positive Gaussian curvature to accommodate the in-plane strain differential (Figure 3B). Unlike photothermal systems, where gels reswell upon removal of light as heat is dissipated, ${ }^{61}$ these gels retain their shape when illumination stops because of the long thermal relaxation time of the cis-isomer, which is $\approx 15 \mathrm{~h}$ at room temperature (Figure S5). Thus, even in the absence of illumination, host-guest complexes remain dissociated following light exposure. However, the gel can be returned to its initial flat state by restoring host-guest interactions via cis-trans photoisomerization with exposure to white light of total intensity $1.2 \mathrm{~W} \mathrm{~cm} \mathrm{Cm}^{-2}$ (with $\approx 100 \mathrm{~mW} \mathrm{~cm} \mathrm{~cm}^{-2}$ across the wavelengths corresponding to the $\mathrm{n}, \pi^{*}$ transition of the cis isomer) for $60 \mathrm{~s}$ to ensure full cis-trans isomerization (Figure $3 \mathrm{C}$ ). Then, the gel can be reprogrammed into another shape by shining a different pattern of UV light. For example, by patterning the gel with localized deswelling in the center of the film, a saddle-like shape with negative Gaussian curvature is formed (Figure 3C). While either type of deformation can easily be achieved with a given sample in previously reported photocross-linkable and photopatternable gel systems, ${ }^{4}$ those platforms are limited to reversible transformations between preprogrammed states. In contrast, the current method allows for multiple shape transformations to be programmed and reprogrammed in a single material without the need for complex chemical reprogramming processes like ionic printing, ${ }^{15}$ nanoparticle reduction, ${ }^{62}$ or acid-base treatment ${ }^{63}$ as reported previously.

In summary, we have developed a simple method to pattern and deploy photoactive gels using reversible host-guest complexes. Specifically, the extent of swelling can be controlled by the isomer population of incorporated azobenzene photoswitches, thereby controlling gel hydrophilicity through transazobenzene complexation with $\alpha$-cyclodextrin. Furthermore, localized deswelling can be exploited to control Gaussian curvature using photolithographic patterning, and a single gel can be rewritten through sequential exposure to white and UV light. While Gaussian curvature was the topic of this study, this strategy could feasibly be extended to control both the Gaussian and mean curvature of a gel simultaneously through judicious selection of light intensity, wavelength, and spatial distribution. Finally, operation at room temperature with biocompatible components makes this materials platform particularly promising for use in biological systems for drug delivery or tissue engineering.

\section{ASSOCIATED CONTENT}

\section{Supporting Information}

The Supporting Information is available free of charge at https://pubs.acs.org/doi/10.1021/acsmacrolett.0c00469.

Synthesis protocol, experimental and characterization methods, and additional UV-vis spectra (PDF)

Video of deswelling of a gel at $22{ }^{\circ} \mathrm{C}$ in $\alpha$-CD solution under flood UV illumination. Playback is at $1 \times$ speed (MP4)

\section{AUTHOR INFORMATION}

\section{Corresponding Authors}

Ryan C. Hayward - Department of Polymer Science and Engineering, University of Massachusetts Amherst, Amherst, Massachusetts 01003, United States; 이이. orcid.org/0000-00016483-2234; Email: hayward@umass.edu

Arri Priimagi - Smart Photonic Materials, Faculty of Engineering and Natural Sciences, Tampere University, Tampere, Finland; ○ orcid.org/0000-0002-5945-9671; Email: arri.priimagi@ tuni.fi

\section{Authors}

Alexa S. Kuenstler - Department of Polymer Science and Engineering, University of Massachusetts Amherst, Amherst, Massachusetts 01003, United States

Markus Lahikainen - Smart Photonic Materials, Faculty of Engineering and Natural Sciences, Tampere University, Tampere, Finland; orcid.org/0000-0002-4891-5352 
Hantao Zhou - Department of Polymer Science and Engineering, University of Massachusetts Amherst, Amherst, Massachusetts 01003, United States

Wenwen Xu - Department of Polymer Science and Engineering, University of Massachusetts Amherst, Amherst, Massachusetts 01003, United States

Complete contact information is available at: https://pubs.acs.org/10.1021/acsmacrolett.0c00469

\section{Author Contributions}

${ }^{\S}$ A.S.K. and M.L. contributed equally

\section{Notes}

The authors declare no competing financial interest.

\section{ACKNOWLEDGMENTS}

Support for this work was provided by the Office of Naval Research through the MURI on Photomechanical Materials (ONR N00014-18-1-2624). A.P. acknowledges the financial support of the European Research Council (Starting Grant Project PHOTOTUNE, 679646). M.L. is thankful for the Emil Aaltonen Foundation for the funding support as well for the International HR Services of Tampere University for funding a 3 month research internship to the University of Massachusetts Amherst. This work is part of the Academy of Finland Flagship Programme (Photonics Research and Innovation, 320165).

\section{REFERENCES}

(1) Brown, T. E.; Anseth, K. S. Spatiotemporal Hydrogel Biomaterials for Regenerative Medicine. Chem. Soc. Rev. 2017, 46 (21), 6532-6552.

(2) Ionov, L. Hydrogel-Based Actuators: Possibilities and Limitations. Mater. Today 2014, 17 (10), 494-503.

(3) Sharon, E.; Efrati, E. The Mechanics of Non-Euclidean Plates. Soft Matter 2010, 6 (22), 5693-5704.

(4) Jeon, S. J.; Hauser, A. W.; Hayward, R. C. Shape-Morphing Materials from Stimuli-Responsive Hydrogel Hybrids. Acc. Chem. Res. 2017, 50 (2), 161-169.

(5) Erol, O.; Pantula, A.; Liu, W.; Gracias, D. H. Transformer Hydrogels: A Review. Adv. Mater. Technol. 2019, 4 (4), 1-27.

(6) Klein, Y.; Efrati, E.; Sharon, E. Shaping of Elastic Sheets by Prescription of Non-Euclidean Metrics. Science 2007, 315 (5815), $1116-1120$.

(7) Wu, Z. L.; Moshe, M.; Greener, J.; Therien-Aubin, H.; Nie, Z.; Sharon, E.; Kumacheva, E. Three-Dimensional Shape Transformations of Hydrogel Sheets Induced by Small-Scale Modulation of Internal Stresses. Nat. Commun. 2013, 4 (1), 1-7.

(8) Kim, J.; Hanna, J. a.; Byun, M.; Santangelo, C. D.; Hayward, R. C. Designing Responsive Buckled Surfaces by Halftone Gel Lithography. Science 2012, 335 (6073), 1201-1205.

(9) Zhou, Y.; Duque, C. M.; Santangelo, C. D.; Hayward, R. C. Biasing Buckling Direction in Shape-Programmable Hydrogel Sheets with Through-Thickness Gradients. Adv. Funct. Mater. 2019, 29 (48), 1905273.

(10) Nojoomi, A.; Arslan, H.; Lee, K.; Yum, K. Bioinspired 3D Structures with Programmable Morphologies and Motions. Nat. Commun. 2018, 9 (1), 1-11.

(11) Sydney Gladman, A.; Matsumoto, E. A.; Nuzzo, R. G.; Mahadevan, L.; Lewis, J. A. Biomimetic 4D Printing. Nat. Mater. 2016, 15 (4), 413-418.

(12) Huang, L.; Jiang, R.; Wu, J.; Song, J.; Bai, H.; Li, B.; Zhao, Q.; Xie, T. Ultrafast Digital Printing toward 4D Shape Changing Materials. Adv. Mater. 2017, 29 (7), 1605390.

(13) Thérien-Aubin, H.; Moshe, M.; Sharon, E.; Kumacheva, E. Shape Transformations of Soft Matter Governed by Bi-Axial Stresses. Soft Matter 2015, 11 (23), 4600-4605.
(14) Wang, Z. J.; Hong, W.; Wu, Z. L.; Zheng, Q. Site-Specific PreSwelling-Directed Morphing Structures of Patterned Hydrogels. Angew. Chem., Int. Ed. 2017, 56 (50), 15974-15978.

(15) Palleau, E.; Morales, D.; Dickey, M. D.; Velev, O. D. Reversible Patterning and Actuation of Hydrogels by Electrically Assisted Ionoprinting. Nat. Commun. 2013, 4 (1), 1-7.

(16) Yu, C.; Duan, Z.; Yuan, P.; Li, Y.; Su, Y.; Zhang, X.; Pan, Y.; Dai, L. L.; Nuzzo, R. G.; Huang, Y.; et al. Electronically Programmable, Reversible Shape Change in Two- and Three-Dimensional Hydrogel Structures. Adv. Mater. 2013, 25 (11), 1541-1546.

(17) Wang, E.; Desai, M. S.; Lee, S. W. Light-Controlled GrapheneElastin Composite Hydrogel Actuators. Nano Lett. 2013, 13 (6), 28262830.

(18) Zhang, X.; Pint, C. L.; Lee, M. H.; Schubert, B. E.; Jamshidi, A.; Takei, K.; Ko, H.; Gillies, A.; Bardhan, R.; Urban, J. J.; et al. Opticallyand Thermally-Responsive Programmable Materials Based on Carbon Nanotube-Hydrogel Polymer Composites. Nano Lett. 2011, 11 (8), 3239-3244.

(19) Ma, C.; Le, X.; Tang, X.; He, J.; Xiao, P.; Zheng, J.; Xiao, H.; Lu, W.; Zhang, J.; Huang, Y.; et al. A Multiresponsive Anisotropic Hydrogel with Macroscopic 3D Complex Deformations. Adv. Funct. Mater. 2016, 26 (47), 8670-8676.

(20) Zhu, Z.; Senses, E.; Akcora, P.; Sukhishvili, S. A. Programmable Light-Controlled Shape Changes in Layered Polymer Nanocomposites. ACS Nano 2012, 6 (4), 3152-3162.

(21) Kim, H.; Kang, J.; Zhou, Y.; Kuenstler, A. S.; Kim, Y.; Chen, C.; Emrick, T.; Hayward, R. C. Light-Driven Shape Morphing, Assembly, and Motion of Nanocomposite Gel Surfers. Adv. Mater. 2019, 31 (27), 1900932.

(22) Mourran, A.; Zhang, H.; Vinokur, R.; Moller, M. Soft Microrobots Employing Nonequilibrium Actuation via Plasmonic Heating. Adv. Mater. 2017, 29 (2), 1604825.

(23) Shi, Q.; Xia, H.; Li, P.; Wang, Y. S.; Wang, L.; Li, S. X.; Wang, G.; Lv, C.; Niu, L. G.; Sun, H. B. Photothermal Surface Plasmon Resonance and Interband Transition-Enhanced Nanocomposite Hydrogel Actuators with Hand-Like Dynamic Manipulation. Adv. Opt. Mater. 2017, 5 (22), 1-9.

(24) Sutton, A.; Shirman, T.; Timonen, J. V. I.; England, G. T.; Kim, P.; Kolle, M.; Ferrante, T.; Zarzar, L. D.; Strong, E.; Aizenberg, J. Photothermally Triggered Actuation of Hybrid Materials as a New Platform for in Vitro Cell Manipulation. Nat. Commun. 2017, 8 (1), 113

(25) Guo, H.; Liu, Y.; Yang, Y.; Wu, G.; Demella, K.; Raghavan, S. R.; Nie, Z. A Shape-Shifting Composite Hydrogel Sheet with Spatially Patterned Plasmonic Nanoparticles. J. Mater. Chem. B 2019, 7 (10), $1679-1683$.

(26) Kuenstler, A. S.; Hayward, R. C. Light-Induced Shape Morphing of Thin Films. Curr. Opin. Colloid Interface Sci. 2019, 40, 70-86.

(27) Kuksenok, O.; Balazs, A. C. Modeling the Photoinduced Reconfiguration and Directed Motion of Polymer Gels. Adv. Funct. Mater. 2013, 23 (36), 4601-4610.

(28) Dehghany, M.; Zhang, H.; Naghdabadi, R.; Hu, Y. A Thermodynamically-Consistent Large Deformation Theory Coupling Photochemical Reaction and Electrochemistry for Light-Responsive Gels. J. Mech. Phys. Solids 2018, 116 (March), 239-266.

(29) Ziółkowski, B.; Florea, L.; Theobald, J.; Benito-Lopez, F.; Diamond, D. Self-Protonating Spiropyran-Co-NIPAM-Co-Acrylic Acid Hydrogel Photoactuators. Soft Matter 2013, 9 (36), 8754-8760.

(30) Stumpel, J. E.; Ziółkowski, B.; Florea, L.; Diamond, D.; Broer, D. J.; Schenning, A. P. H. J. Photoswitchable Ratchet Surface Topographies Based on Self-Protonating Spiropyran-NIPAAM Hydrogels. ACS Appl. Mater. Interfaces 2014, 6 (10), 7268-7274.

(31) Satoh, T.; Sumaru, K.; Takagi, T.; Kanamori, T. Fast-Reversible Light-Driven Hydrogels Consisting of Spirobenzopyran-Functionalized Poly(N-Isopropylacrylamide). Soft Matter 2011, 7 (18), 80308034.

(32) Li, C.; Iscen, A.; Palmer, L. C.; Schatz, G. C.; Stupp, S. I. LightDriven Expansion of Spiropyran Hydrogels. J. Am. Chem. Soc. 2020, 142 (18), 8447-8453. 
(33) Bandara, H. M. D.; Burdette, S. C. Photoisomerization in Different Classes of Azobenzene. Chem. Soc. Rev. 2012, 41 (5), 18091825.

(34) Ube, T.; Ikeda, T. Photomobile Polymer Materials with Complex 3D Deformation, Continuous Motions, Self-Regulation, and Enhanced Processability. Adv. Opt. Mater. 2019, 7 (16), 1900380.

(35) White, T. J.; Broer, D. J. Programmable and Adaptive Mechanics with Liquid Crystal Polymer Networks and Elastomers. Nat. Mater. 2015, 14 (11), 1087-1098.

(36) Matsubara, K.; Watanabe, M.; Takeoka, Y. A Thermally Adjustable Multicolor Photochromic Hydrogel. Angew. Chem., Int. Ed. 2007, 46 (10), 1688-1692.

(37) Kamenjicki, M.; Lednev, I. K.; Mikhonin, A.; Kesavamoorthy, R.; Asher, S. A. Photochemically Controlled Photonic Crystals. Adv. Funct. Mater. 2003, 13 (10), 774-780.

(38) Zhang, Q. M.; Li, X.; Islam, M. R.; Wei, M.; Serpe, M. J. Light Switchable Optical Materials from Azobenzene Crosslinked Poly(NIsopropylacrylamide)-Based Microgels. J. Mater. Chem. C 2014, 2 (34), 6961-6965.

(39) Jiang, W.; Wang, G.; He, Y.; Wang, X.; An, Y.; Song, Y.; Jiang, L. Photo-Switched Wettability on an Electrostatic Self-Assembly Azobenzene Monolayer. Chem. Commun. 2005, 28, 3550-3552.

(40) Kang, M. S.; Gupta, V. K. Photochromic Cross-Links in Thermoresponsive Hydrogels of Poly(N-Isopropylacrylamide): Enthalpic and Entropic Consequences on Swelling Behavior. J. Phys. Chem. B 2002, 106 (16), 4127-4132.

(41) Mudiyanselage, T. K.; Neckers, D. C. Photochromic Superabsorbent Polymers. Soft Matter 2008, 4 (4), 768-774.

(42) Wang, D.; Zhao, W.; Wei, Q.; Zhao, C.; Zheng, Y. Photoswitchable Azobenzene/Cyclodextrin Host-Guest Complexes: From UV- to Visible/Near-IR-Light-Responsive Systems. ChemPhotoChem. 2018, 2 (5), 403-415.

(43) Harada, A.; Takashima, Y.; Nakahata, M. Supramolecular Polymeric Materials via Cyclodextrin-Guest Interactions. Acc. Chem. Res. 2014, 47 (7), 2128-2140.

(44) Sakai, T.; Murayama, H.; Nagano, S.; Takeoka, Y.; Kidowaki, M.; Ito, K.; Seki, T. Photoresponsive Slide-Ring Gel. Adv. Mater. 2007, 19 (15), 2023-2025.

(45) Takashima, Y.; Hatanaka, S.; Otsubo, M.; Nakahata, M.; Kakuta, T.; Hashidzume, A.; Yamaguchi, H.; Harada, A. Expansion-Contraction of Photoresponsive Artificial Muscle Regulated by Host-Guest Interactions. Nat. Commun. 2012, 3 (1), 1-8.

(46) Zheng, Z.; Hu, J.; Wang, H.; Huang, J.; Yu, Y.; Zhang, Q.; Cheng, Y. Dynamic Softening or Stiffening a Supramolecular Hydrogel by Ultraviolet or Near-Infrared Light. ACS Appl. Mater. Interfaces 2017, 9 (29), 24511-24517.

(47) Zhao, Y.-L.; Stoddart, J. F. Azobenzene-Based Light-Responsive Hydrogel System. Langmuir 2009, 25 (15), 8442-8446.

(48) Tamesue, S.; Takashima, Y.; Yamaguchi, H.; Shinkai, S.; Harada, A. Photoswitchable Supramolecular Hydrogels Formed by Cyclodextrins and Azobenzene Polymers. Angew. Chem., Int. Ed. 2010, 49 (41), 7461-7464.

(49) Schmidt, B. V. K. J.; Hetzer, M.; Ritter, H.; Barner-Kowollik, C. Modulation of the Thermoresponsive Behavior of $\operatorname{Poly}(\mathrm{N}, \mathrm{N}$ Diethylacrylamide) via Cyclodextrin Host/Guest Interactions. Macromol. Rapid Commun. 2013, 34 (16), 1306-1311.

(50) Yamaguchi, H.; Kobayashi, Y.; Kobayashi, R.; Takashima, Y.; Hashidzume, A.; Harada, A. Photoswitchable Gel Assembly Based on Molecular Recognition. Nat. Commun. 2012, 3 (1), 1-5.

(51) Wang, M.; Zhang, X.; Li, L.; Wang, J.; Wang, J.; Ma, J.; Yuan, Z.; Lincoln, S. F.; Guo, X. Photo-Reversible Supramolecular Hydrogels Assembled by $\alpha$-Cyclodextrin and Azobenzene Substituted Poly(Acrylic Acid)s: Effect of Substitution Degree, Concentration, and Tethered Chain Length. Macromol. Mater. Eng. 2016, 301 (2), 191198.

(52) Yoon, J.; Cai, S.; Suo, Z.; Hayward, R. C. Poroelastic Swelling Kinetics of Thin Hydrogel Layers: Comparison of Theory and Experiment. Soft Matter 2010, 6 (23), 6004-6012.
(53) Otake, K.; Inomata, H.; Konno, M.; Saito, S. Thermal Analysis of the Volume Phase Transition with N-Isopropylacrylamide Gels. Macromolecules 1990, 23 (1), 283-289.

(54) Wan, P.; Jiang, Y.; Wang, Y.; Wang, Z.; Zhang, X. Tuning Surface Wettability through Photocontrolled Reversible Molecular Shuttle. Chem. Commun. 2008, No. 44, 5710-5712.

(55) Roy, D.; Brooks, W. L. A.; Sumerlin, B. S. New Directions in Thermoresponsive Polymers. Chem. Soc. Rev. 2013, 42 (17), 72147243.

(56) Luo, C.; Zuo, F.; Zheng, Z.; Ding, X.; Peng, Y. Temperature/ Light Dual-Responsive Inclusion Complexes of $\alpha$-Cyclodextrins and Azobenzene-Containing Polymers. J. Macromol. Sci., Part A: Pure Appl.Chem. 2008, 45 (5), 364-371.

(57) Kungwatchakun, D.; Irie, M. Photoresponsive Polymers. Photocontrol of the Phase Separation Temperature of Aqueous Solutions of Poly-[N-isopropylacrylamide-co-N-(4-phenylazophenyl)Acrylamide]. Makromol. Chem., Rapid Commun. 1988, 9 (4), 243-246.

(58) Ritter, H.; Sadowski, O.; Tepper, E. Influence of Cyclodextrin Molecules on the Synthesis and the Thermoresponsive Solution Behavior of N-Isopropylacrylamide Copolymers with Adamantyl Groups in the Side-Chains. Angew. Chem., Int. Ed. 2003, 42 (27), $3171-3173$.

(59) Lee, K. M.; White, T. J. Photochemical Mechanism and Photothermal Considerations in the Mechanical Response of Monodomain, Azobenzene-Functionalized Liquid Crystal Polymer Networks. Macromolecules 2012, 45 (17), 7163-7170.

(60) Lahikainen, M.; Zeng, H.; Priimagi, A. Reconfigurable Photoactuator through Synergistic Use of Photochemical and Photothermal Effects. Nat. Commun. 2018, 9 (1), 1-8.

(61) Hauser, A. W.; Evans, A. A.; Na, J. H.; Hayward, R. C. Photothermally Reprogrammable Buckling of Nanocomposite Gel Sheets. Angew. Chem., Int. Ed. 2015, 54 (18), 5434-5437.

(62) Guo, H.; Cheng, J.; Yang, K.; Demella, K.; Li, T.; Raghavan, S. R.; Nie, Z. Programming the Shape Transformation of a Composite Hydrogel Sheet via Erasable and Rewritable Nanoparticle Patterns. ACS Appl. Mater. Interfaces 2019, 11 (45), 42654-42660.

(63) Kohlmeyer, R. R.; Buskohl, P. R.; Deneault, J. R.; Durstock, M. F.; Vaia, R. A.; Chen, J. Shape-Reprogrammable Polymers: Encoding, Erasing, and Re-Encoding. Adv. Mater. 2014, 26 (48), 8114-8119. 